\title{
Review of cancer treatment with immune checkpoint inhibitors
}

\author{
Current concepts, expectations, limitations and pitfalls
}

\author{
Christiane Thallinger (D) - Thorsten Füreder · Matthias Preusser · Gerwin Heller · Leonhard Müllauer • \\ Christoph Höller · Helmut Prosch · Natalija Frank · Rafal Swierzewski · Walter Berger · Ulrich Jäger · \\ Christoph Zielinski
}

Received: 22 August 2017 / Accepted: 10 October 2017 / Published online: 2 November 2017 (C) The Author(s) 2017. This article is an open access publication.

\begin{abstract}
Summary Immunotherapy by checkpoint inhibition is about to profoundly change cancer therapy. The number of indications are growing at an unprecedented speed. Clinical studies have demonstrated efficacy in a variety of solid tumors and in hematologic malignancies, although some clinical trials have produced negative results. Thus, it is fair to assume that there are obvious limitations and pitfalls in immunotherapy. Future concepts for combination treatment of immune checkpoint inhibitors have to be developed, but there is also urgent need for better and standardized biomarkers to identify those cancer patients who will benefit from treatment by checkpoint inhibition. The current overview summarizes current knowledge on immune checkpoint inhibitor treatment in malignancies, its outlook and limitations, diagnostic means and, finally, side effect management.
\end{abstract}

Priv. Doz. Dr. C. Thallinger, MBA, MSc $(\bowtie) \cdot$ T. Füreder • M. Preusser · G. Heller · C. Zielinski

Clinical Division of Oncology, Department of Medicine I,

General Hospital, Währinger Gürtel 18-20, 1090 Vienna, Austria

christiane.thallinger@meduniwien.ac.at

Priv. Doz. Dr. C. Thallinger, MBA, MSc · C. Zielinski

Central European Cooperative Oncology Group, Vienna, Austria

Priv. Doz. Dr. C. Thallinger, MBA, MSc · T. Füreder · M. Preusser · G. Heller · N. Frank · C. Zielinski

Comprehensive Cancer Center, General Hospital, Medical University Vienna, Vienna, Austria

\section{Müllauer}

Department of Pathology, General Hospital, Medical

University Vienna, Vienna, Austria
Keywords Cancer · Immunotherapy · PD-1 · Experts · Review

\section{Introduction}

In the treatment of solid malignancies, immune checkpoint inhibitors constitute an important breakthrough positively influencing treatment outcomes regarding progression-free (PFS) and/or overall survival (OS), as compared to chemotherapy-based treatment. Immune checkpoint inhibitor treatment involves antibodies generated against the cytotoxic T-lymphocyte associated protein 4 (CTLA-4), the programmed death receptor 1 (PD-1) or its ligand (PD-L1). Thus, immune checkpoint inhibitors modulate the interaction between tumor cells and cytotoxic T lymphocytes in the tumor environment, which are exhausted in their

\section{Höller}

Department of Dermatology, General Hospital, Medical University Vienna, Vienna, Austria

\section{H. Prosch}

Department of Radiology, General Hospital, Medical University Vienna, Vienna, Austria

R. Swierzewski

European Cancer Patient Coalition (ECPC), Brussels, Belgium

W. Berger

Institute for Cancer Research, Department of Medicine I, Medical University Vienna, Vienna, Austria

U. Jäger

Clinical Division of Hematology, Department of Medicine I,

Medical University Vienna, Vienna, Austria 
function. Targeting with CTLA-4 or PD-1 or PD-L1 reverses the exhaustion of cytotoxic $\mathrm{T}$ lymphocytes thus leading to the elimination of tumor cells via the re-induction of the "natural" function of the $\mathrm{T}$ cell population. Interestingly, some of the clinical results when using anti PD-1 and anti PD-L1 antibodies may be also due to additional effects on T-cells including their targeting of B7.1 [1-4].

Whereas anti-CTLA-4 antibodies (ipilimumab and tremelimumab), anti-PD-1 antibodies (nivolumab and pembrolizumab), and anti-PD-L1 antibodies (atezolizumab, avelumab and durvalumab) have produced remarkable results regarding tumor control in many malignancies (albeit in various treatment lines), response is often followed by relapse and disease progression. Conversely, cancer patients who benefit from checkpoint inhibition can achieve longterm benefit and remarkable duration of PFS. Inhibition of CTLA-4 by ipilimumab represents the first compound ever used in immune checkpoint inhibitor treatment to enter clinical routine. Ipilimumab was licensed for use in advanced metastatic melanoma [5]. The second group of drugs for immune checkpoint inhibition were anti PD-1 or anti PD-L1 antibodies which are currently registered by the U.S. Food and Drug Administration (FDA) for metastatic malignant melanoma, non-small cell lung cancer (NSCLC), renal cell cancer (RCC), head and neck cancer (HNSC), urothelial carcinoma and Hodgkin's lymphoma in various stages of the respective disease and in the context of varying treatment histories [6-12]. Many other malignancies (e.g. hepatocellular carcinoma, ovarian cancer, mesothelioma, gastric cancer, B-cell non-Hodgkin lymphoma) are currently under clinical investigation to determine a possible efficacy of checkpoint inhibition [13-15]. Landmark trials have shown significant improvement of survival rates in many advanced metastatic cancers (e.g. NSCLC, HNSC, metastatic malignant melanoma and renal cell carcinoma), when compared with chemotherapy or, as in RCC,other treatment modalities, such as everolimus in second line.

\section{Current indications for immune checkpoint in- hibitor treatment}

Indications for immune checkpoint inhibitor treatment are rapidly increasing [16]: while PD-1 and CTLA-4 directed antibodies were primarily registered for the treatment of metastatic malignant melanoma, NSCLC followed quickly with currently nivolumab, pembrolizumab and atezolizumab licensed for second line treatment, and pembrolizumab for first line treatment for patients with NSCLC and PD-L1 expression of $>50 \%$. Additional indications currently comprise renal and urothelial cancer, HNSC squamous epithelial cancer and Hodgkin's lymphoma.

\section{Hematologic disorders}

In hematology, the checkpoint inhibitors nivolumab and pembrolizumab have been approved for the treatment of Hodgkin's disease [17]: Classical Hodgkin's lymphoma cells show high PD-L1 expression. Clinical studies have been primarily performed in patient groups with relapsed disease after autologous stem cell transplantation ineligible for autologous stem cell transplantation, a relapse after brentuximab vedotin therapy or without this treatment. The outcome was remarkable $[18,19]$ : overall response rates (ORR) were up to $65 \%$ and complete remission (CR) occurred in approximately $20 \%$ of patients. Preliminary results from a phase 1 and 2 study of brentuximab vedotin in combination with nivolumab in patients with relapsed or refractory Hodgkin's lymphoma showed ORR of $90 \%(26 / 29)$ and CR of $62 \%(18 / 29)$.

Many clinical studies with checkpoint inhibitors are currently under way to test their efficacy in various other hematologic indications either as monotherapy or, as in the case of multiple myeloma, in combination with immunomodulators, such as lenalidomide and pomalidomide [20].

Further concepts applied in the field of hematologic malignancies look at the combination of immune checkpoint inhibitors with other immunotherapies: primarily, combinations with bispecific T-cell engaging antibody (BITE/blinatumomab) or with chimeric antigen receptor $\mathrm{T}$ cells (CAR-T-cells) [21]. Although both treatments depend on functional T-cells, immune checkpoint inhibitors could enhance the T-cell response to make BITE or CAR-T-cell therapy more effective [11, 22, 23]. In childhood relapsed or refractory acute lymphocytic leukemia, the combination of CAR-T-cell immunotherapy and checkpoint inhibition showed an overall remission rate of $82 \%$ within 3 months and an OS of $89 \%$ at 6 months.

\section{Predictive biomarkers}

For anti CTLA-4 therapy, some biomarkers have been identified which may be useful for predictive purposes, but none has entered clinical routine or should be used before starting treatment.

\section{$P D-L 1$}

For anti-PD1 and anti-PD-L1 therapies, mainly immune cell-associated PD-L1 expression has been identified to represent an important predictive enrichment biomarker. Although widely accepted, many important questions remain open regarding the generalization of PD-L1 being a reliable biomarker: thus, PD-L1 expression is induced by cytokines therefore generating only a "snapshot" of the overall biological situation [24]. As a further limitation, various cut-off values of PD-L1 expression have been used in various clinical trials varying between 1, 5, 10, 25 and 50\%. 
Considering the current state of knowledge it seems acceptable to generalize, however, that higher PDL1 values might indicate a better response to therapy leading to the fact that PD-L1 is currently accepted as the best available enrichment biomarker [25, 26]. In addition, other immune-related biomarkers are also currently being studied and may be of predictive importance, which mainly include intratumoral CD8+ T-cell infiltration as a manifestation of the immunogenicity of a certain tumor [27].

\section{Mutational load and molecular alterations}

Another group of predictive biomarkers could be derived from the assessment of the "mutational landscape" (mutational load) of a tumor: this assumption was first corroborated by an early publication on the efficacy of nivolumab in NSCLC, and more recently in a study with pembrolizumab in NSCLC with more than 200 mutations in protein-encoding regions of the tumor genome [28]; however, this field is evolving relatively slowly, and there is no established cut-off value, which would be clinically applicable [29, 30].

In a related context and regarding mismatch repair-deficient cancers, microsatellite instability (MSIH) could predict response to checkpoint inhibition therapy in colorectal cancer [31]. Some $15 \%$ of colorectal cancers and some $22 \%$ of endometrial and gastric carcinomas show MSI-H frequencies of $>10 \%$. These tests might, therefore, be of value in treatment decisions for the use of immune checkpoint inhibitor treatment in cancers of the gastrointestinal tract [32]. At present, PD-L1 immunohistochemistry and MSI status assessed in all gastrointestinal cancers by immunohistochemistry or by molecular MSI are used as biomarkers in the current clinical setting, while mutations in DNA polymerase genes (POLE, POLD1) could become predictive biomarkers in endometrial and colon cancer in the future [33, 34].

Similarly, the loss of DNA methylation of certain genes including the cancer testis antigens (CTAs) and their consequent upregulation in tumor cells (e.g. MAGE-A1, MAGE-A3, NY-ESO-1, SSX-2, SSX-4) might indicate a better response to immune checkpoint blockade, as these antigens may be recognized as non-self structures [35-37].

On another note, hyperprogression, which is found rarely but repeatedly during treatment with immune checkpoint inhibitors might be correlated with MDM2/MDM4 amplification, EGFR alterations and/or $D N M T 3 A$ mutations, which might lead to early recognition, if properly validated.

\section{Radiologic investigations in immune checkpoint inhibitor treatment}

Radiologically, tumor size is the easiest parameter for the determination on an effect of any anticancer treatment. Although this also applies to treatment with im- mune checkpoint inhibitors, there are particular treatment responses, which are not observed in patients receiving other kind of treatments. As immunotherapies do not target directly the tumor cells but the immune system, the time to a measurable tumor response can be variable. Therefore, in some patients the tumors may remain stable in size or even grow slowly over some weeks or even months before they show a decrease in size. In other patients, the infiltration of tumors by inflammatory cells leads to a temporary increase in tumor size. This so-called pseudoprogession is observed in $10-15 \%$ of patients with melanomas and in less than $2 \%$ of patients with lung cancer and must not be confused with treatment failure. In analogy, even new lesions might become visible during immune checkpoint blockade, which could be the result of an ensuing visibility of previously undetected metastases due to lymphocyte infiltration. To discriminate progressive disease from pseudoprogression, short-term follow-up examinations not earlier than 4 weeks after the examination in which a progression of disease was observed are advised. Thus, the course of disease under treatment with immune checkpoint inhibitors should be monitored by comparing serial and repeated measurement of target lesions before treatment is abandoned, always keeping in mind the biological principles of treatment versus tumor control. This, however, only applies to patients with no clinical deterioration.

For study purposes, a number of response criteria have been developed, the first one being the immunerelated response criteria (irRC) published in 2009 [38]. Very recently, new immune response criteria in solid tumors (iRECIST) criteria have been published, which will be used in future prospective trials in addition to conventional response criteria [39, 40].

\section{Adverse events and side effects management}

Immunotherapy by checkpoint inhibition can cause immune-related adverse events (irAEs) in a considerable number of patients due to the induction of overstimulation of immune reactivity or to the generation of outright autoimmune phenomena [41]. With CTLA-4-inhibition, such side effects are observed in up to 7 patients out of 10 , while with PD-(L) 1 inhibitor treatment, these occur in only 2-3 out of 10 [42]. As immunotherapy in cancer is assumed to activate the tumor-directed T-cell response by T-cells infiltrating the primary tumor and its metastases, this therapeutic intervention can also cause irAEs in all types of tissues. These irAEs may include the induction of diarrhea or the emergence of ulcerative colitis or Crohn's disease, Hashimoto's thyroiditis, autoimmune hepatitis, uveitis and hypophysitis which can be life-threatening complications if not recognized and treated appropriately [41, 43, 44].

With rash and pruritus often occurring as the first side effect of anti CTLA-4 treatment, liver toxicity, di- 
arrhea, colitis and hypophysitis tend to appear later. In PD-(L) 1 inhibition, most common irAEs are cutaneous and gastrointestinal, less common endocrine, hepatic, pulmonary and renal. Combination of checkpoint inhibitors and duration of therapy cause more severe adverse events typically associated with those encountered during CTLA-4 immune checkpoint inhibition.

In patients receiving immune checkpoint inhibition treatment, every symptom has to be suspected to represent a sign of a possible irAE, and patients should be informed that they should contact the hospital once a possible side effect occurs. Similarly, the patients' general practitioners should have basic information about irAEs. Early diagnosis and onset of treatment can prevent the development from grades 1-2 to grades 4-5 toxicities. At the hospital, an interdisciplinary team should be ready to assess and manage side effects of immunotherapy according to published management algorithms. While grade 1, irAEs should be managed symptomatically under continued PD-(L) 1 inhibition, grades 2 and 3 toxicities necessitate delay of treatment plus the addition of 1-2 mg prednisone/ $\mathrm{kg}$ body weight with tapering to a dosage of below $10 \mathrm{mg}$. Grade 4 events and recurrent grade 3 events should cause permanent discontinuation of PD-(L) 1 inhibition $[45,46]$.

\section{Overcoming treatment resistance}

When following patients under immune checkpoint inhibitor treatment, it became clear that primary and adaptive resistance to immunotherapy might occur which limits the efficacy of treatment. This calls for concepts to maximize the clinical benefit of immune checkpoint inhibitor treatment by the combination with either other immunotherapy, with chemotherapy, with radiotherapy or with targeted therapies using tyrosine kinase inhibitors. For all of these therapeutic approaches, an abundance of clinical trials in a similar abundance of clinical scenarios and diseases are under way. Aims are to not only increase the activation and function of immune cell-associated tumor cell destruction, but also to widen the current concept to other immune cells including $\mathrm{T}$ regulatory cells, myeloid-derived suppressor cells and neutrophils, and, finally, make tumors more immunogenic and induce their infiltration by immunocompetent cells. Overcoming resistance mechanisms will be the key for the obtainment of an enhanced efficacy of immunotherapy in cancer [47].

Primary and intrinsic mechanisms of resistance or mechanisms of adaptation as well as secondary or acquired resistance are the limiting factors for immune checkpoint inhibitor treatment. Thus, gene editing with amplification of PD-L1 in malignant cells appears to occur early and makes malignant cells prone to respond to PD-(L) 1 inhibitors, whereas the loss of PDL1 seems to be the cause of resistance to treatment occurring in some patients. Thus, future strategies should not only address the malignant cells themselves but also their microenvironment and the function of immunocompetent cells $[48,49]$. These strategies are given here:

\section{Combinations of immune checkpoint inhibitor treatment with other immunotherapies}

The idea of addressing the immune response and the tumor microenvironment has been studied in the Checkmate 012 study in first line treatment of stage IV NSCLC, as CTLA-4 inhibition by ipilimumab is directed at the microenvironment, which should increase the efficacy of nivolumab seen in this disease [51]. The proof of this concept was generated by the fact that in a subgroup of patients, a partial response was observed in $39 \%$ with PFS at 24 weeks seen in $63 \%$ of patients; however, an abundance of other immunomodulating compounds, such as vaccines in combination with immune checkpoint inhibitors and antagonistic antibodies directed against proteins, such as LAG-3 or activating antibodies against such peptides as OX-40 are in current clinical testing [52].

\section{Combinations of immune checkpoint inhibitors with chemotherapy}

Checkpoint inhibition plus chemotherapy may show additional benefits as cytotoxic drugs have more effects than only causing death of malignant cells thereby setting free cancer-associated antigens but also by interfering with the function of a series of immunocompetent cells. Thus and as examples, anthracyclines augment dendritic cell activation, cyclophosphamide promotes anti-tumor CD4+ cells and cell recognition and lysis by CD8+ cells, and cisplatin abrogates the activity of T-regulatory cells and myeloid-derived suppressor cells [53-55].

\section{Combination of immune checkpoint inhibitors with tyrosine kinase inhibitors}

In the combination of targeted therapies with immune checkpoint inhibition, early studies using ipilimumab and vemurafenib have shown that care has to be taken regarding potential severe toxicities, particularly hepatotoxicity [56]. This seems to depend on the drugs of choice and the sequence of their application as, for an impressive example, the combination of nivolumab with VEGF inhibitors or TKIs may decrease the number and function of T-regulatory cells. The disruption of angiogenesis under the mentioned treatment plus immune checkpoint inhibition has shown to exert important efficacy in the treatment of advanced RCC. 


\section{Combination of immune checkpoint inhibitors with radiotherapy}

An abundance of clinical trials are under way do determine the effect of a combination of checkpoint inhibitors with radiotherapy which have been primarily observed by the induction of an abscopal effect resulting from antigen exposure originating from destroyed cancer cells and the induction of a type I IFN response $[57,58]$.

\section{Limitations to access}

The actual status and future perspectives of immunotherapy in cancer depend on the access of patients to these innovative treatments. Even in the most affluent member states of the EU, there are considerable differences in this field. With currently $28 \mathrm{EU}$ member states and their 28 various healthcare and reimbursement systems and 28 various interpretations of the EU Cross-border Directive make access to checkpoint inhibitor treatment complex and diverse. New pricing models could be a future way for reimbursement in order to simplify the access to this important class of drugs [59-61]. The involvement of patients and patient advocacy groups must become the norm in HTA assessment which should serve the patients to get access to innovative drugs, as outlined in the magnitude of clinical benefit scale generated by the European Society for Medical Oncology [62].

Acknowledgements The authors are grateful to Mr. Wolfgang Wagner for the generation of the current manuscript and to Bristol Myers-Squibb for the provision of an educational grant to the Central European Cooperative Study Group (CECOG) to make the educational activity possible.

Funding Open access funding provided by Medical University of Vienna.

Conflict of interest C. Thallinger, T. Füreder, M. Preusser, G. Heller, L. Müllauer, C. Höller, H. Prosch, N. Frank, R. Swierzewski, W. Berger, U. Jäger, and C. Zielinski received speakers fees from CECOG for this meeting.

Open Access This article is distributed under the terms of the Creative Commons Attribution 4.0 International License (http://creativecommons.org/licenses/by/4.0/), which permits unrestricted use, distribution, and reproduction in any medium, provided you give appropriate credit to the original author(s) and the source, provide a link to the Creative Commons license, and indicate if changes were made.

\section{References}

1. Aydin AM, Woldu SL, Hutchinson RC, Boegemann M, Bagrodia A, Lotan Y, Margulis V, Krabbe LM. Spotlight on atezolizumab and its potential in the treatment of advanced urothelial bladder cancer. Onco Targets Ther. 2017;10:1487-502.

2. Balar AV, Galsky MD, Rosenberg JE, Powles T, Petrylak DP, Bellmunt J, Loriot Y, Necchi A, Hoffman-Censits J, PerezGracia JL, et al. Atezolizumab as first-line treatment in cisplatin-ineligible patients with locally advanced and metastatic urothelial carcinoma: a single-arm, multicentre, phase 2 trial. Lancet. 2017;389(10064):67-76.

3. Duraiswamy J, Freeman GJ, Coukos G. Therapeutic PD1 pathway blockade augments with other modalities of immunotherapyT-cell function to preventimmune decline in ovarian cancer. Cancer Res. 2013;73(23):6900-12.

4. Kumar R, Yu F, Zhen YH, Li B, Wang J, Yang Y, Ge HX, Hu PS, Xiu J. PD-1 blockade restores impaired function of ex vivo expanded CD8+ T cells and enhances apoptosis in mismatch repair deficient EpCAM+PD-L1+ cancer cells. Onco Targets Ther. 2017;10:3453-65.

5. Hodi FS, O'Day SJ, McDermott DF, Weber RW, Sosman JA, Haanen JB, Gonzalez R, RobertC, SchadendorfD, Hassel JC, et al. Improved survival with ipilimumab in patients with metastatic melanoma. NEngl J Med. 2010;363(8):711-23.

6. Allen PB, Gordon LI. PD-1 blockade in Hodgkin's lymphoma: learning new tricks from an old teacher. Expert Rev Hematol. 2016;9(10):939-49.

7. Beckermann KE, Johnson DB, Sosman JA. PD-1/PD-L1 blockade in renal cell cancer. Expert Rev Clin Immunol. 2017;13(1):77-84.

8. Beckermann KE, Jolly PC, Kim JY, Bordeaux J, Puzanov I, Rathmell WK, Johnson DB. Clinical and immunologic correlates of response to PD-1 blockade in a patient with metastatic renal medullary carcinoma. J Immunother Cancer. 2017;5:1.

9. GaronEB. Current perspectives in immunotherapy for nonsmall cell lung cancer. Semin Oncol. 2015;42(Suppl 2):S11-S8.

10. Garon EB. Selecting patients for immune checkpoint inhibition in lung cancer. Clin Adv Hematol Oncol. 2015;13(8):490-2.

11. Robert C, Schachter J, Long GV, Arance A, Grob JJ, Mortier L, Daud A, Carlino MS, McNeil C, Lotem M, et al. Pembrolizumab versus Ipilimumab in advanced melanoma. NEngl J Med. 2015;372(26):2521-32.

12. Sonpavde G. PD-1 and PD-L1 inhibitors as salvage therapy for urothelial carcinoma. $\mathrm{N}$ Engl J Med. 2017;376(11):1073-4.

13. Gaillard SL, Secord AA, Monk B. The role of immune checkpoint inhibition in the treatment of ovarian cancer. Gynecol OncolRes Pract. 2016;24(3):11.

14. Khanna S, Thomas A, Abate-Daga D, Zhang J, Morrow B, Steinberg SM, Orlandi A, Ferroni P, Schlom J, Guadagni F, et al. Malignant mesothelioma effusions are infiltrated by CD3+ T cells highly expressing PD-L1 and the PD-L1+ tumor cells within these effusions are susceptible to ADCC by the anti-PD-L1 antibody avelumab. J Thorac Oncol. 2016;11(11):1993-2005.

15. LordickF, Shitara K, Janjigian YY. New agents on the horizon in gastric cancer. Ann Oncol. 2017;28(8):1767-75.

16. Procaccio L, Schirripa M, Fassan M, Vecchione L, Bergamo F, Prete AA, Intini R, Manai C, Dadduzio V, Boscolo A, et al. Immunotherapy in gastrointestinal cancers. Biomed Res Int. 2017; https:// doi.org/10.1155/2017/4346576.

17. Ilcus C, Bagacean C, Tempescul A, Popescu C, Parvu A, Cenariu M, Bocsan C, Zdrenghea M. Immune checkpoint blockade: the role of PD-1-PD-L axis in lymphoid malignancies. Onco Targets Ther. 2017;10:2349-63.

18. Armand P, Shipp MA, Ribrag V, Michot JM, Zinzani PL, Kuruvilla J, Snyder ES, Ricart AD, Balakumaran A, Rose S, et al. Programmed death-1 blockade with pembrolizumabin patients with classical Hodgkin Lymphoma after brentuximab vedotin failure. J Clin Oncol. 2016;34(31):3733-9. https:// doi.org/10.1200/JCO.2016.67.3467. 
19. Zinzani PL, Ribrag V, Moskowitz CH, Michot JM, Kuruvilla J, Balakumaran A, Zhang Y, Chlosta S, Shipp MA, Armand P. Safety and tolerability of pembrolizumab in patients with relapsed/refractory primary mediastinal large B-cell lymphoma. Blood. 2017;130(3):267-70.

20. Larocca A, Mina R, Gay F, Bringhen S, Boccadoro M. Emerging drugs and combinations to treat multiple myeloma. Oncotarget. 2017;8(36):60656-72.

21. FribergG,BlinatumomabRD. (Blincyto(R)); lessonslearned from the bispecific $t$-cell engager $(\operatorname{BiTE}(\mathrm{R}))$ in acute lymphocyticleukemia (ALL).Ann Oncol. 2017;1;28(8):2009-12. Aug.

22. Hartmann J, Schussler-Lenz M, Bondanza A, Buchholz CJ. Clinical development of CAR T cells-challenges and opportunities in translatinginnovative treatment concepts. EMBO Mol Med. 2017;9(9):1183-97.

23. Romero D. Haematological cancer: after ibrutinib, CAR T cellsinduceresponses. NatRevClin Oncol. 2017;14(10):588.

24. WangW, Shen G, Wu S, SongS, NiY, SuoZ, MengX, Li D, Zhou L, Hao R, et al. PD-1 mRNA expression in peripheral blood cells and its modulation characteristics in cancer patients. Oncotarget. 2017;8(31):50782-91.

25. Dai C, Wang M, Lu J, Dai Z, Lin S, Yang P, Tian T, Liu X, Min W, DaiZ. Prognostic and predictive values of PD-L1 expression in patients with digestive system cancer: a meta-analysis. Onco Targets Ther. 2017;10:3625-34.

26. Kitano A, Ono M, Yoshida M, Noguchi E, Shimomura A, Shimoi T, Kodaira M, Yunokawa M, Yonemori K, Shimizu C, et al. Tumour-infiltrating lymphocytes are correlated with higher expression levels of PD-1 and PD-L1 in early breast cancer. ESMO Open. 2017;2(2):e150.

27. Teng MW, Ngiow SF, Ribas A, Smyth MJ. Classifying cancers based on T-cell infiltration and PD-L1. Cancer Res. 2015;75(11):2139-45.

28. Rizvi NA, Hellmann MD, Snyder A, Kvistborg P, Makarov V, Havel JJ, Lee W, Yuan J, Wong P, Ho TS, et al. ancer immunology. Mutational landscape determines sensitivity to PD-1 blockade in non-small cell lung cancer. Science. 2015;348(6230):124-8.

29. Anagnostou V, Smith KN, Forde PM, Niknafs N, Bhattacharya R, White J, Zhang T, Adleff V, Phallen J, Wali N, et al. Evolution of neoantigen landscape during immune checkpoint blockade in non-small cell lung cancer. Cancer Discov. 2017;7(3):264-76.

30. Roszik J, Haydu LE, Hess KR, Oba J, Joon AY, Siroy AE, Karpinets TV, Stingo FC, Baladandayuthapani V, Tetzlaff MT, et al. Novel algorithmic approach predicts tumor mutation load and correlates with immunotherapy clinical outcomes using a defined gene mutation set. BMC Med. 2016;14(1):168

31. Kwon Y, Park M, Jang M, Yun S, Kim WK, Kim S, Paik S, Lee HJ, Hong S, Kim TI, et al. Prognosis of stage III colorectal carcinomas with FOLFOX adjuvant chemotherapy can be predicted by molecular subtype. Oncotarget. 2017;8(24):39367-81.

32. Sohn BH, Hwang JE, Jang HJ, Lee HS, Oh SC, Shim JJ, Lee KW, Kim EH, Yim SY, Lee SH, et al. Clinical significance of four molecular subtypes of gastric cancer identified by the Cancer Genome Atlas Project. Clin Cancer Res. 2017; https://doi.org/10.1158/1078-0432.CCR-16-2211.

33. Ahn SM, Ansari AA, Kim J, Kim D, Chun SM, Kim J, Kim TW, Park I, Yu CS, Jang SJ. The somatic POLE P286R mutation defines a unique subclass of colorectal cancer featuring hypermutation, representing a potential genomic biomarker for immunotherapy. Oncotarget. 2016;7(42):68638-49.

34. Van Gool IC, Eggink FA, Freeman-Mills L, Stelloo E, Marchi E, de Bruyn M, Palles C, Nout RA, de Kroon CD, Osse EM, et al. POLE proofreading mutations elicit an antitumor immune response in endometrial cancer. Clin Cancer Res. 2015;21(14):3347-55.

35. Laban S, Giebel G, Klumper N, Schrock A, Doescher J, Spagnoli G, Thierauf J, Theodoraki MN, Remark R, Gnjatic $\mathrm{S}$, et al. MAGE expression in head and neck squamous cell carcinoma primary tumors, lymph node metastases and respective recurrences-implications for immunotherapy. Oncotarget. 2017;8(9):14719-35.

36. Li J, Zou X, Li C, Zhong J, Chen Y, Zhang X, Qi F, Li M, Cai Z, Tang A. Expression of novel cancer/testis antigen TMEM31 increases during metastatic melanoma progression. Oncol Lett. 2017;13(4):2269-73.

37. Zajac P, Schultz-Thater E, Tornillo L, Sadowski C, Trella E, Mengus C, Iezzi G, Spagnoli GC. MAGE-A antigens and cancer immunotherapy. Front Med (Lausanne). 2017;4:18.

38. Wolchok JD, Hoos A, O'Day S, Weber JS, Hamid O, Lebbe C, Maio M, Binder M, Bohnsack O, Nichol G, et al. Guidelines for the evaluation of immune therapy activity in solid tumors: immune-related response criteria. Clin Cancer Res. 2009;15(23):7412-20.

39. Litiere S, Collette S, de Vries EG, Seymour L, Bogaerts J. RECIST-learning from the past to build the future. Nat Rev Clin Oncol. 2017;14(3):187-92.

40. Seymour L, Bogaerts J, Perrone A, Ford R, Schwartz LH, Mandrekar S, Lin NU, Litiere S, Dancey J, Chen A, et al. iRECIST: guidelines for response criteria for use in trials testing immunotherapeutics. Lancet Oncol. 2017;18(3):e143-e52.

41. Michot JM, Bigenwald C, Champiat S, Collins M, Carbonnel F, Postel-Vinay S, Berdelou A, Varga A, Bahleda R, Hollebecque $A$, et al. Immune-related adverse events with immune checkpoint blockade: a comprehensive review. Eur JCancer. 2016;54:139-48.

42. De Velasco G, Je Y, Bosse D, Awad MM, Ott PA, Moreira RB, Schutz F, Bellmunt J, Sonpavde GP, Hodi FS, et al. Comprehensive meta-analysis of key immune-related adverse events from CTLA-4 and PD-1/PD-L1 inhibitors in cancer patients. Cancer Immunol Res. 2017;5(4):312-8.

43. Bertrand A, Kostine M, Barnetche T, Truchetet ME, Schaeverbeke T. Immune related adverse events associated with anti-CTLA-4 antibodies: systematic review and meta-analysis. BMCMed. 2015; 13:211.

44. Friedman CF, Snyder A. Atypical autoimmune adverse effects with checkpoint blockade therapies. Ann Oncol. 2017;28(2):206-7.

45. Friedman CF, Proverbs-Singh TA, Postow MA. Treatment of the immune-related adverse effects of immune checkpoint inhibitors: a review. JAMA Oncol. 2016;2(10):1346-53.

46. Hellmann MD, Friedman CF, Wolchok JD. Combinatorial cancer immunotherapies. Adv Immunol. 2016;130:251-77.

47. Pulluri B, Kumar A, Shaheen M, Jeter J, Sundararajan S. Tumor microenvironment changes leading to resistance of immune checkpoint inhibitors in metastatic melanoma and strategies to overcome resistance. Pharmacol Res. 2017;123:95-102.

48. Hahn AW, Gill DM, Pal SK, Agarwal N. The future of immune checkpoint cancer therapy after PD- 1 and CTLA- 4 . Immunotherapy. 2017;9(8):681-92.

49. Calvo E, Schmidinger M, Heng DY, Grunwald V, Escudier B. Improvement in survival end points of patients with metastaticrenalcellcarcinomathroughsequential targeted therapy. Cancer Treat Rev. 2016;50:109-17.

50. Hammers HJ, Plimack ER, Infante JR, Rini BI, McDermott DF, Lewis LD, Voss MH, Sharma P, Pal SK, Razak ARA, et al. Safety and efficacy of Nivolumab in combination with Ipilimumab in metastatic renal cell carcinoma: the 
CheckMate 016 study. J Clin Oncol. 2017; https://doi.org/ 10.1200/JCO.2016.72.1985.

51. Hellmann MD, Rizvi NA, Goldman JW, Gettinger SN, Borghaei H, Brahmer JR, Ready NE, Gerber DE, Chow LQ, Juergens RA, et al. Nivolumab plus ipilimumab as first-line treatmentfor advanced non-small-cell lung cancer (CheckMate 012): results of an open-label, phase 1, multicohort study. Lancet Oncol. 2017;18(1):31-41.

52. Granier C, De Guillebon E, Blanc C, Roussel H, Badoual C, Colin E, Saldmann A, Gey A, Oudard S, Tartour E. Mechanisms of action and rationale for the use of checkpoint inhibitors in cancer. ESMO Open. 2017;2(2):e213.

53. Chen C, ChenZ, Chen D, ZhangB, WangZ, LeH. Suppressive effects of gemcitabine plus cisplatin chemotherapy on regulatory $\mathrm{T}$ cells in nonsmall-cell lung cancer. J Int Med Res. 2015;43(2):180-7.

54. GhiringhelliF, MenardC,PuigPE,LadoireS, RouxS, MartinF, SolaryE,LeCesneA,ZitvogelL, ChauffertB. Metronomiccyclophosphamide regimen selectively depletes CD4+CD25+ regulatory $\mathrm{T}$ cells and restores $\mathrm{T}$ and $\mathrm{NK}$ effector functions in end stage cancer patients. Cancer Immunol Immunother. 2007;56(5):641-8.

55. Vacchelli E, Ma Y, Baracco EE, Sistigu A, Enot DP, Pietrocola F, Yang H, Adjemian S, Chaba K, Semeraro M, et al. Chemotherapy-induced antitumor immunity requires formyl peptide receptor 1. Science. 2015;350(6263):972-8.

56. Ribas A, Hodi FS, Callahan M, Konto C, Wolchok J. Hepatotoxicity with combination of vemurafenib and ipilimumab. NEngl J Med. 2013;368(14):1365-6.
57. Thallinger C, Prager G, Ringl H, Zielinski C. Abscopal effect in the treatment of malignant melanoma. Hautarzt. 2015;66(7):545-8.

58. Weichselbaum RR, Liang H, Deng L, Fu YX. Radiotherapy and immunotherapy: a beneficial liaison? Nat Rev Clin Oncol. 2017;14(6):365-79.

59. Dimitrova N, Znaor A, Agius D, Eser S, Sekerija M, RyzhovA, Primic-Zakelj M, Coebergh JW, Group SEEW. Breast cancer in South-Eastern European countries since 2000: rising incidence and decreasing mortality at young and middle ages. Eur J Cancer. 2017;83:43-55.

60. Kandolf Sekulovic L, Peris K, Hauschild A, Stratigos A, Grob JJ, Nathan P, Dummer R, Forsea AM, Hoeller C, Gogas H, et al. More than 5000 patients with metastatic melanoma in Europe per year do not have access to recommended firstline innovative treatments. Eur J Cancer. 2017;75:313-22.

61. Mihajlovic J, Dolk C, Tolley K, Simoens S, Postma MJ. Reimbursement of targeted cancer therapies within 3 different European health care systems. Clin Ther. 2015;37(2):474-80.

62. Kiesewetter B, Raderer M, Prager GW, Fuereder T, Marosi C, Preusser M, Krainer M, Locker GJ, Brodowicz T, Zielinski CC. The European Society for Medical Oncology "Magnitude of Clinical Benefit Scale" field-tested in infrequent tumour entities: an extended analysis of its feasibility at the Medical University of Vienna. ESMO Open. 2017;2(3):e166. 